\title{
Fixed-targets Serial crystallography at SPring-8 and SACLA
}

\author{
Masaki Yamamoto*1, Kazuya Hasegawa ${ }^{1,2}$, Keitaro Yamashita ${ }^{1,3}$, Seiki Baba ${ }^{1,2}$, Kunio Hirata ${ }^{1}$, \\ Go Ueno $^{1}$, Hideo $\mathrm{Ago}^{1}$, Takashi Kumasaka ${ }^{1,2}$ \\ 1 RIKEN SPring-8 Center, 2 JASRI SPring-8, 3 The University of Tokyo \\ *yamamoto@riken.jp
}

The serial femtosecond crystallography (SFX) developed at XFELs changed the convention of MX data collection; more than ten thousand of images are collected from multiple micro-crystals delivered on the X-ray beam, and the data are processed by Monte Carlo integration. The success of SFX motivated researchers to establish serial crystallography using synchrotron micro-focus beamlines (serial synchrotron crystallography; SSX) by combining a high-intensity microbeam and a fast-readout detector. At SPring- 8 campus, we have been engaged in the development of serial crystallography by using both SPring- 8 micro-focus beamlines and XFEL SACLA (SPring-8 Angstrom Compact free-electron LAser).

At SACLA, de novo phasing of Hg-bound luciferin-regenerating enzyme (Hg-LRE) was performed by using SFX; firstly we solved the structure by SIR-AS[1], but lately succeeded in SAD phasing [2]. The Hg-LRE was also used for the establishment of the fixed-targets serial synchrotron rotation crystallography (SSROX) at BL41XU following the protocol of Gati et al. [3] ; diffraction images are collected from a large number of micro-crystals frozen in a cryoloop by combining 2D raster scan and goniometer rotation [4]. This study revealed that less than 1,000 images were sufficient for the successful SAD phasing in SSROX, which means that rotation is effective in SSX. Now, room temperature SSROX is examined aiming at future development of time resolved crystallography using pink beam at BL41XU.

We will present experimental results and comparisons of SFX and SSX in order to discuss the future prospect of MX.

\section{References:}

[1] Yamashita et al., (2015). Sci Rep. 5, 14017

[2] Yamashita et al., (2017). IUCrJ. 4, 639-647

[3] Gati, C. et al. (2014). IUCrJ. 1, 87-94.

[4] Hasegawa. K. et al. (2017). J. Synchotron Rad. 24, 29-41. 